\title{
A clinicopathological case report of retinopathy of pancreatitis
}

\author{
MARILYN C. KINCAID, W. RICHARD GREEN, DAVID L. KNOX, AND \\ CHARLES MOHLER \\ From the Eye Pathology Laboratory, the Wilmer Ophthalmological Institute, and \\ Department of Pathology, the Johns Hopkins Medical Institutions, Baltimore, Maryland, USA
}

\begin{abstract}
SUMMARY A 32-year-old black woman with pancreatitis developed visual loss in both eyes. On ophthalmoscopic examination she had extensive ischaemic infarcts at the posterior poles of both eyes, a clinical picture which has been reported previously in patients with pancreatitis and noted to be similar to Purtscher's retinopathy. On histopathological examination of the eyes after death there were arteriolar occlusions in both the choroid and retina and inner ischaemic infarctions with inner retinal oedema. Owing to the 23-day interval from arteriolar occlusion until death the exact nature of the original occlusion could not be determined. However, either fat emboli or activated complement $\mathrm{C} 5$-induced granulocyte embolus formation are possible causes.
\end{abstract}

Purtscher ${ }^{1}$ first described in 1910 the retinopathy that now bears his name. It occurs after trauma to the head ${ }^{1}$ or the body ${ }^{2}$ and is characterised by visual loss within hours and the appearance of cotton-wool spots and haemorrhages at the posterior pole. More recently a similar fundus picture has been recognised in pancreatitis. ${ }^{34}$ The pathogenesis remains obscure. Some have theorised that fat emboli play a causative role in some cases of Purtscher's retinopathy ${ }^{5}$ and in the retinopathy of pancreatitis, ${ }^{3}$ but others ${ }^{6}$ dispute this view. Kelley ${ }^{7}$ believed, on the basis of capillary leakage seen on fluorescein angiography performed within hours of injury, that primary endothelial damage was a more likely cause. However, he could not rule out microemboli.

More recently the role of granulocyte emboli formed by complement activation, specifically C5-induced granulocytic clumping, has been proposed as the aetiology in both Purtscher's retinopathy and in the retinopathy of pancreatitis. ${ }^{4}$

\section{Case report}

The patient was a 32-year-old chronic alcoholic woman. She was admitted to the medical service on 21 May 1972 with epigastric pain and a 3-day history

Correspondence to W. Richard Green, MD, Eye Pathology Laboratory, Johns Hopkins Hospital, $600 \mathrm{~N}$. Wolfe Street, Baltimore, MD 21205, USA. of anorexia. At the time of admission the liver was tender and palpable $3 \mathrm{~cm}$ below the right costal margin. There were skin ecchymoses, and a nasogastric tube drained guaiac-positive coffeeground material. Her blood pressure was $160 / 90$ $\mathrm{mmHg}$. Platelet count, white cell count, and clotting times were normal. Haematocrit was $30 \%$. Amylase was 208 CRW units (normal $=20-160$ ). Haemoglobin electrophoresis was normal.

On the evening of 22 May she had a tonic-clonic seizure and became comatose for 3 days. A neurological examination was normal except for the altered mental status. Arteriogram and echoencephalogram were normal, but the cerebrospinal fluid contained an elevated protein level of $118 \mathrm{mg} / \mathrm{dl}(1.18 \mathrm{~g} / \mathrm{l})$ (normal=15-45 $(0 \cdot 15-0.45 \mathrm{~g} / \mathrm{l})$ ), with a normal glucose level. The neurologist noted 'occasional' dot haemorrhages, with retinal oedema in both eyes. The amylase rose to $372 \mathrm{CRW}$ units, and she developed a fever, for which she was given kanamycin and chloramphenicol.

On 24 May 1972 she was seen by one of us (D.L.K.) and found to have diffuse intra- and subretinal oedema, with narrowed retinal arterioles in both eyes. There were a few retinal haemorrhages. This was thought to be consistent with hypertensive retinopathy. Because of her obtunded state her vision could not be tested.

Her amylase continued to rise, up to 720 units. Calcium was low at $5 \mathrm{mg} / \mathrm{dl}(1.25 \mathrm{mmol} / \mathrm{l})($ normal $=$ 


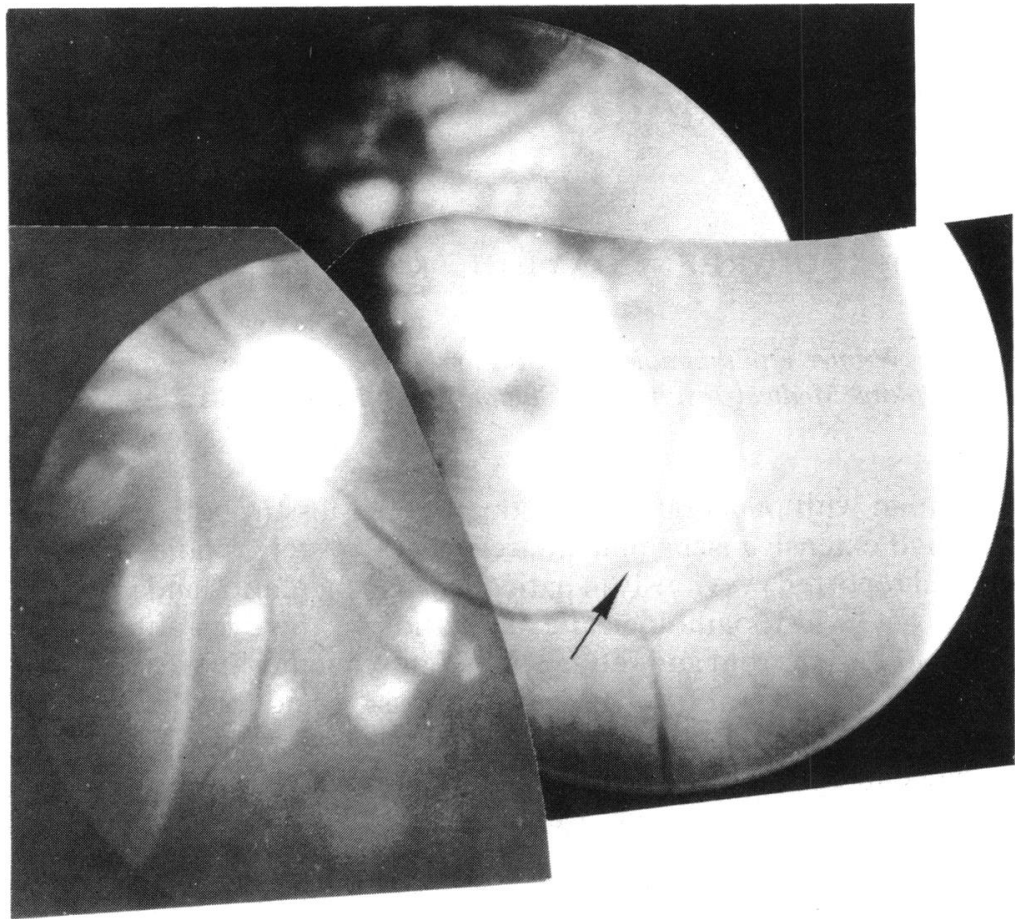

Fig. 1 Composite photograph of left eye taken at the bedside. The macula appears as a cherry-red spot, and fluffy-white exudates are seen throughout the posterior pole. The arterioles (arrow) are narrowed.

9-11 (2.25-2.75 mmol/l)), and albumin was low at $1 \cdot 7 \mathrm{~g} / \mathrm{dl}(17 \mathrm{~g} / \mathrm{l})$ (normal=3.5-5.5 (35-55 g/l)). However, as the amylase level fell, her mental status improved.

Because of a low haematocrit she was transfused, but developed pulmonary oedema, responsive only to repeated phlebotomies. On 26 May 1972 Pseudomonas aeruginosa was grown from her lungs. She continued to have intermittent gastric bleeding, with a drop in the haematocrit. Gastroscopy disclosed massive gastritis.

On 1 June 1972 funduscopic examination by one of us (D.L.K.) revealed pallor and oedema, narrowed arterioles, and subretinal dense white exudate, thought possibly to be lipid. The pupils were reactive but sluggish.

The following day another ophthalmologist saw the patient. He described the deposition of a 'white opalescent coagulum' within the sensory retina in the posterior pole. Arteriolar narrowing was still apparent, and because of the retinal oedema the macula appeared as a cherry-red spot (Fig. 1). He believed that this was a form of lipaemia retinalis consistent with hyperlipidaemia secondary to her pancreatitis, along with hypertensive small-vessel disease. However, although the cholesterol level was $467 \mathrm{mg} / \mathrm{dl}$ (12 mmol/l) on the day of admission, two days later it had dropped to $187 \mathrm{mg} / \mathrm{dl}(4.8 \mathrm{mmol} / \mathrm{l})$, within the normal range, and it remained normal. The triglyceride level was $140 \mathrm{mg} / \mathrm{dl}(1.6 \mathrm{mmol} / \mathrm{l})$, also normal.

As the pancreatitis resolved and the amylase and calcium levels fell towards normal her major problem continued to be bleeding from the gastrointestinal tract. Both prothrombin time and partial thromboplastin time remained prolonged, and she required repeated transfusions. Gastroscopy showed a possible specific lesion on the lesser curvature; so on 11 June she underwent a total gastrectomy, vagotomy, and oesophagojejunostomy. Postoperatively she became oliguric, with rising blood urea nitrogen and creatinine. Since the haematocrit had again fallen, she was re-explored surgically the following day, but no bleeding lesion was found. She was given kanamycin and penicillin for possible sepsis, and was dialysed peritoneally. On 14 June she developed pulmonary oedema, which improved with phlebotomy. However, she became unresponsive with multiple organ failures and died on 15 June 1972.

Necropsy disclosed pancreatitis with fat necrosis of the body and tail of the pancreas, massive gastrointestinal haemorrhage, and necrotising haemorrhagic bronchopneumonia consistent with the clinically observed pseudomonas infection. The kidneys showed hyaline scarring and interstitial fibrosis consistent with hypertension. The brain 


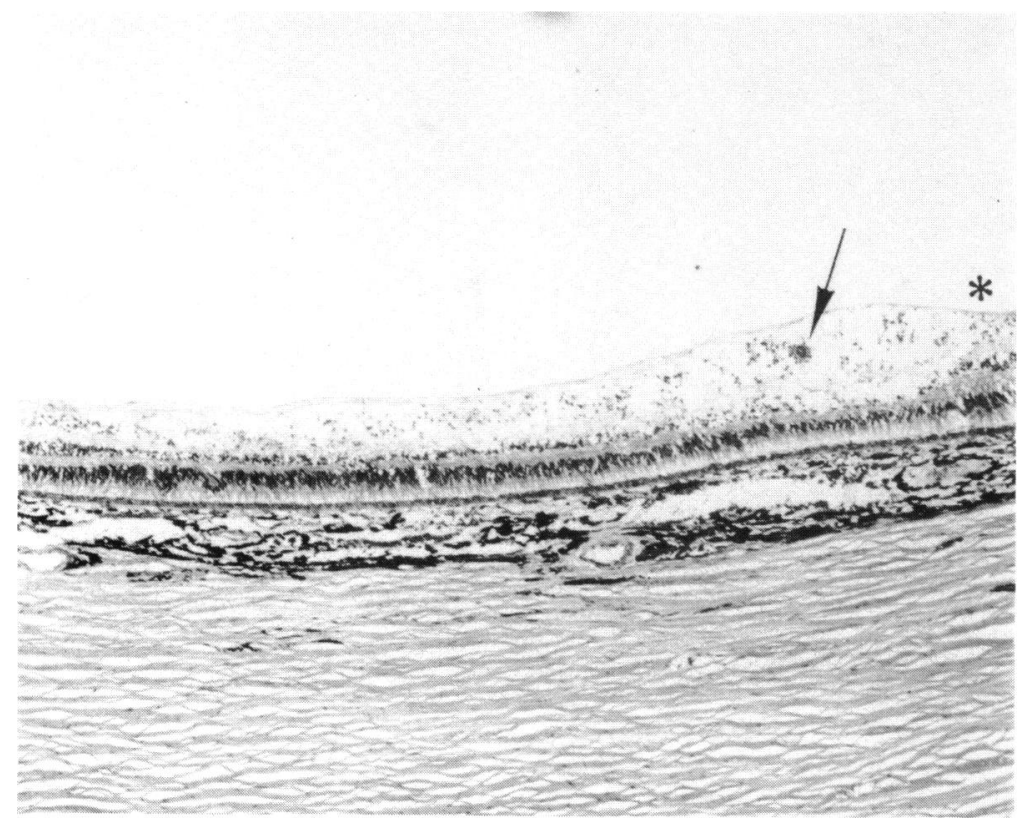

Fig. 2A Low-power view of the left eye showing diffuse oedema extending throughout inner retina. There is partial loss of the nerve fibre, ganglion cell, and inner plexiform layers and inner portion of the inner nuclear layer. The area of transition from normal retina is fairly abrupt (asterisk). An occluded vessel (arrow) is present. (Haematoxylin and eosin, $\times 57$ ).

contained small haemorrhages in the cortex and also the right hippocampus, with bacteria in the necrotic vessel walls. The brain lesions were thought to be similar to those of the lung and could have arisen in a single embolic episode. However, they were believed to be too recent to explain the original neurological symptoms. There was no definite evidence of pseudomonas lesions elsewhere.

\section{Material and methods}

Both eyes were obtained at necropsy and were routinely fixed in a buffered solution of $4 \%$ formaldehyde for several days. They were then washed in water and dissected in $60 \%$ alcohol. The external gross appearance was unremarkable and the eyes were of normal size. They were opened

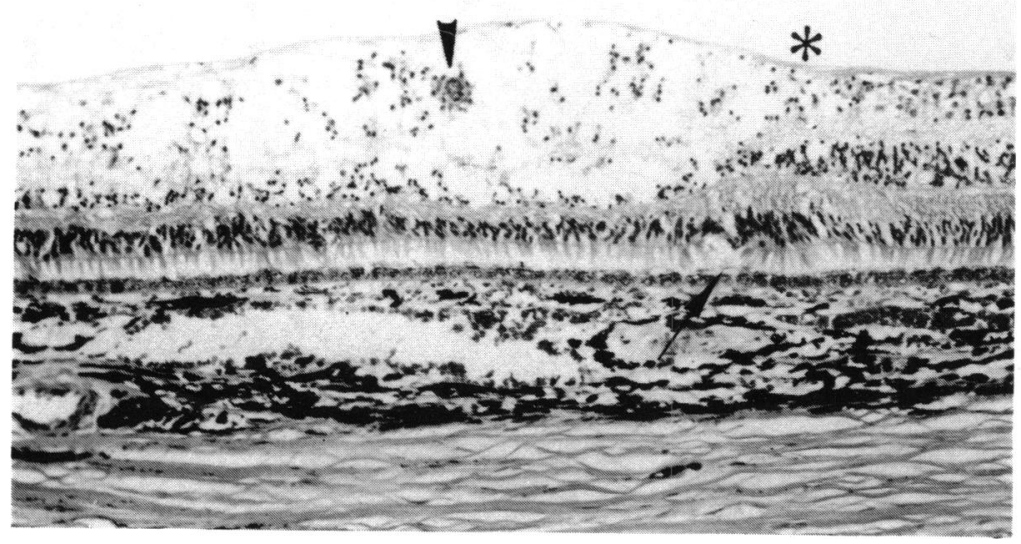

Fig. 2B Higher power view of the same area showing the abrupt transition (asterisk) from normal to oedematous retina. An occluded arteriole (arrow head) is present. $A$ focal area of photoreceptor disruption (arrow) overlying apparently normal RPE is present. (Haematoxylin and eosin, $\times 109$ ). 
Fig. 3A Full thickness of retina of the right eye with inner retinal oedema and also disruption and loss of photoreceptors. There is outer segment (asterisk) and inner segment (arrowheads) loss. The underlying RPE and choroid appear normal. An occluded retinal arteriole (arrow) is present. (Haematoxylin and eosin, $\times 120$ ).

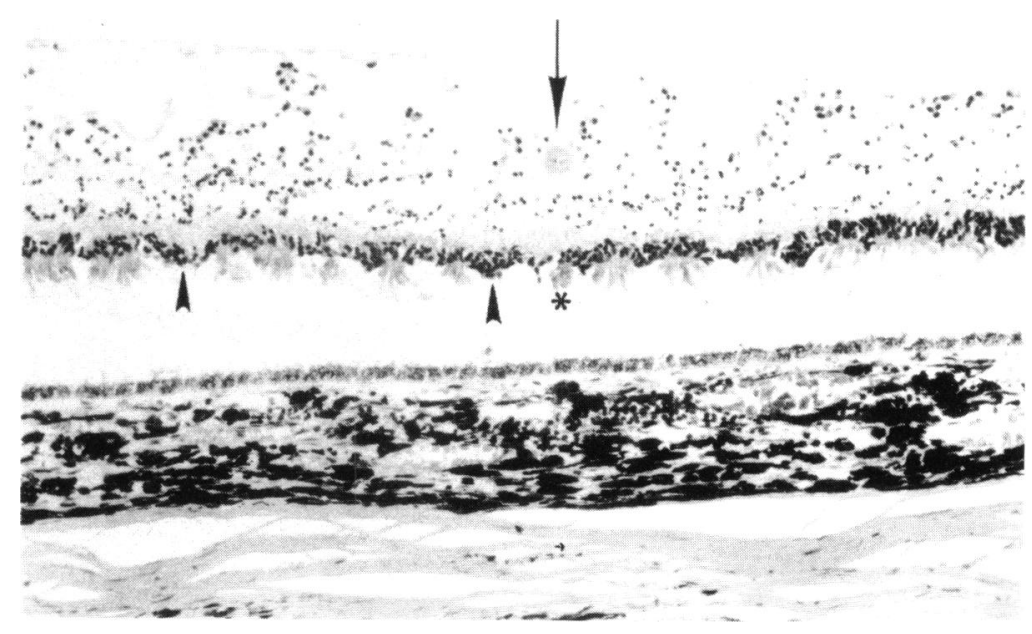

horizontally, and whitish material, thought to be exudate, was present in the posterior poles of both eyes. The pupil-optic nerve portions were then routinely dehydrated and embedded in paraffin, and $0.1 \mathrm{~mm}$ stepped sections taken for light microscopy.

In each eye, within the caps, areas of retinal vascular occlusion could be identified grossly. Therefore, portions of retina which included the vascular occlusions were dissected from the caps and refixed in a buffered $2.5 \%$ glutaraldehydeformaldehyde solution, rinsed in phosphate buffer, postfixed in $2 \%$ osmium tetroxide, dehydrated with graded acetone solutions, and embedded in Epon/ Araldite for transmission electron microscopy. The remainder of the retina from the caps was trypsindigested to study the vasculature.
Fig. 3B Occluded retinal arteriole (arrow) within an area of oedematous inner retina. There is also disruption and loss of photoreceptors (asterisk). (Haematoxylin and eosin, $\times 356)$.

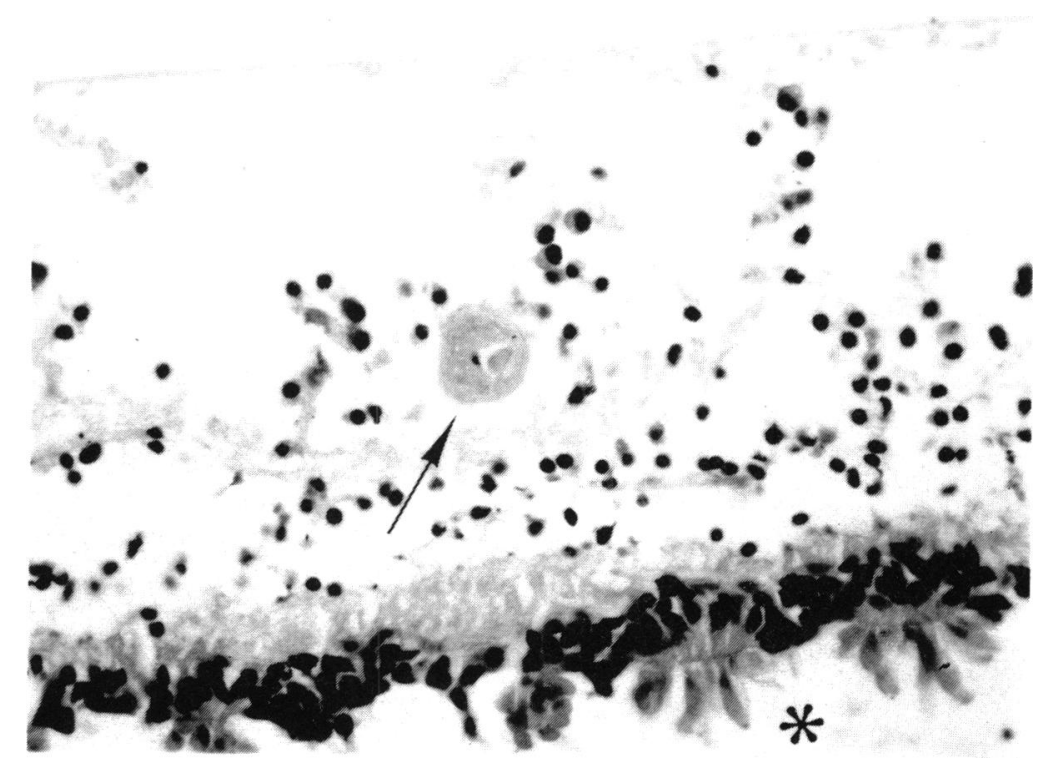




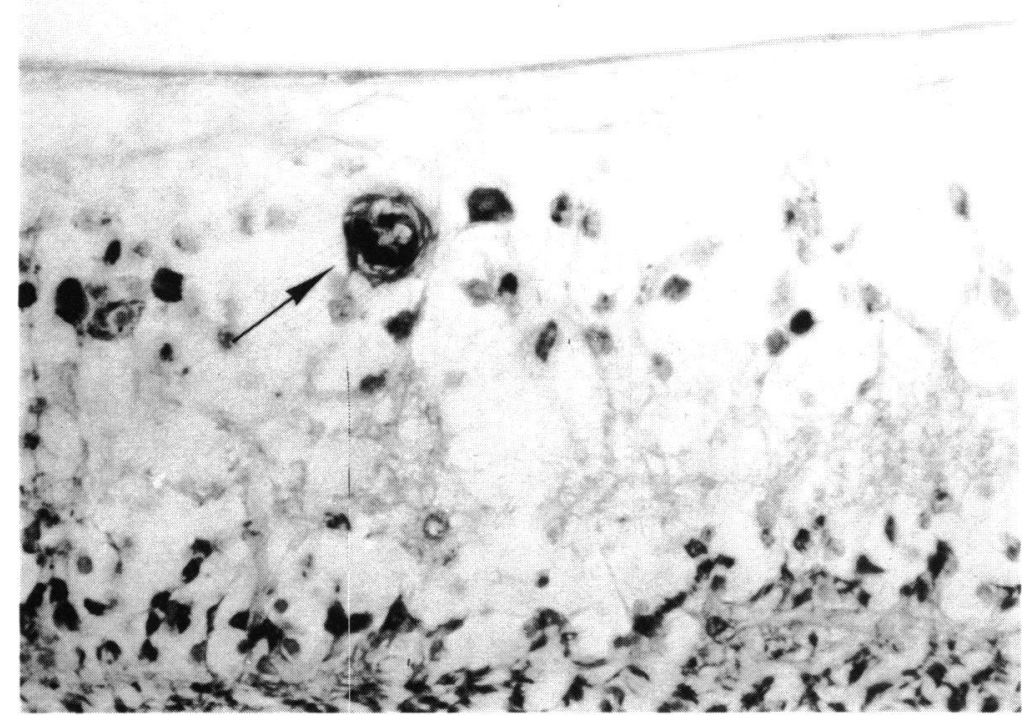

Fig. 3C Another area of retina showing an arteriole completely occluded by fibrin. (Phosphotungstic acid-haematoxvlin, $\times 471$ ).

\section{Results}

Both eyes showed similar changes. The anterior segments were normal except for some red blood cells in the anterior chamber of the right eye. The peripheral retina of both eyes showed typical peripheral cystoid degeneration. Posteriorly there were focal areas of oedema within the inner layers of the retina, with cystoid spaces and disruption of the normal architecture (Fig. 2). Transition from normal to oedematous retina was abrupt. In some areas there was also disruption of the photoreceptors, with loss of photoreceptor segments (Fig. 3). In these areas outer segments, and to a less extent, inner segments were missing, with the remaining photoreceptors displaying a curious bunching together at the outer nuclear layer. However, the underlying retinal pigment epithelium (RPE) and choroid remained normal. In both eyes occluded retinal arterioles were present (Fig. 3B). The occluding material was periodic-acid-Schiff positive. A special stain for fibrin was positive (Fig. 3C). Small haemorrhages were present in the outer plexiform layer (Fig. 4). No occlusions were seen in the material prepared by trypsin digestion. At other levels occluded choroidal vessels were evident which also contained fibrin (Fig. 5).

Electron micrographs revealed small arterioles with narrowed lumina containing proteinaceous material centrally, consistent with recanalised thrombi.

\section{Discussion}

This patient presented with a fundus picture classic for Purtscher's retinopathy, with cotton-wool spots and inner retinal oedema centred round the optic nerve and extending to the macular area. ${ }^{2}$ This fundus appearance has been described in acute pancreatitis also, ${ }^{34}$ but no histopathologic correlation has been available in cases of pancreatitis.

The histopathological findings in our patient support the clinical picture. The inner retinal oedema was widespread, with abrupt transition from normal to oedematous retina. In much of the involved area there were striking changes in the photoreceptor cells. We suggest that the effect of inner retinal distension is to cause the outer retinal layers to become bunched and squeezed together. Such distension might therefore account in part for the changes seen in the photoreceptors. Impairment of choroidal circulation alone was probably not responsible, as the retinal pigment epithelial cells remained normal.

Unfortunately, because of the severity of her illness and altered mental state, the patient's vision was not tested nor could a fluorescein angiogram be done. It seems evident, however, that the retinopathy and possibly the neurological findings were caused by her pancreatitis and not, for instance, due to emboli from the pseudomonas infection of the lung, which occurred much later in the disease course.

It is possible to separate Purtscher's retinopathy 
Fig. 4 A haemorrhage (asterisk) in the outer plexiform layer of the retina of the left eve. (Haematoxylin and eosin, $\times 284$ ).

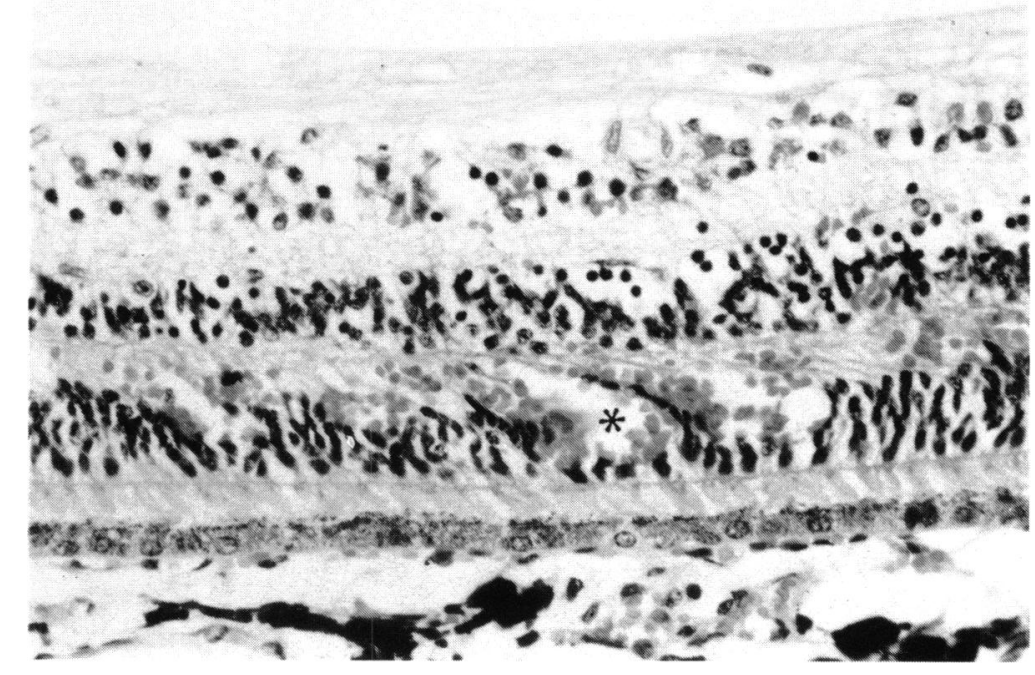

into 2 types in accordance with aetiology: retinopathy caused by fat emboli, and retinopathy caused by sudden elevation in the intravascular pressure.

The first histological evidence for fat emboli causing Purtscher's retinopathy was provided by Urbanek $^{5}$ in 1934. Seven of his patients had longbone fractures and developed retinopathy. $\mathrm{He}$ obtained the eyes from 3 of them and demonstrated emboli in the retina and choroid which histologically were positive for fat.

Since then, there have been other case reports ${ }^{8-10}$ in patients with multiple injuries. These 3 reports all describe patients with extensive injuries and multiple fractures. Examination of the eyes and other body tissues with special stains post mortem disclosed numerous fat emboli throughout. Fat embolism can

Fig. 5 Occluded choroidal artery (arrow) in the right eye. The occluding material is fibrin. The overlying retinal pigment epithelium appears normal. (Phosphotungstic acid-haematoxylin, $\times 400$ ).

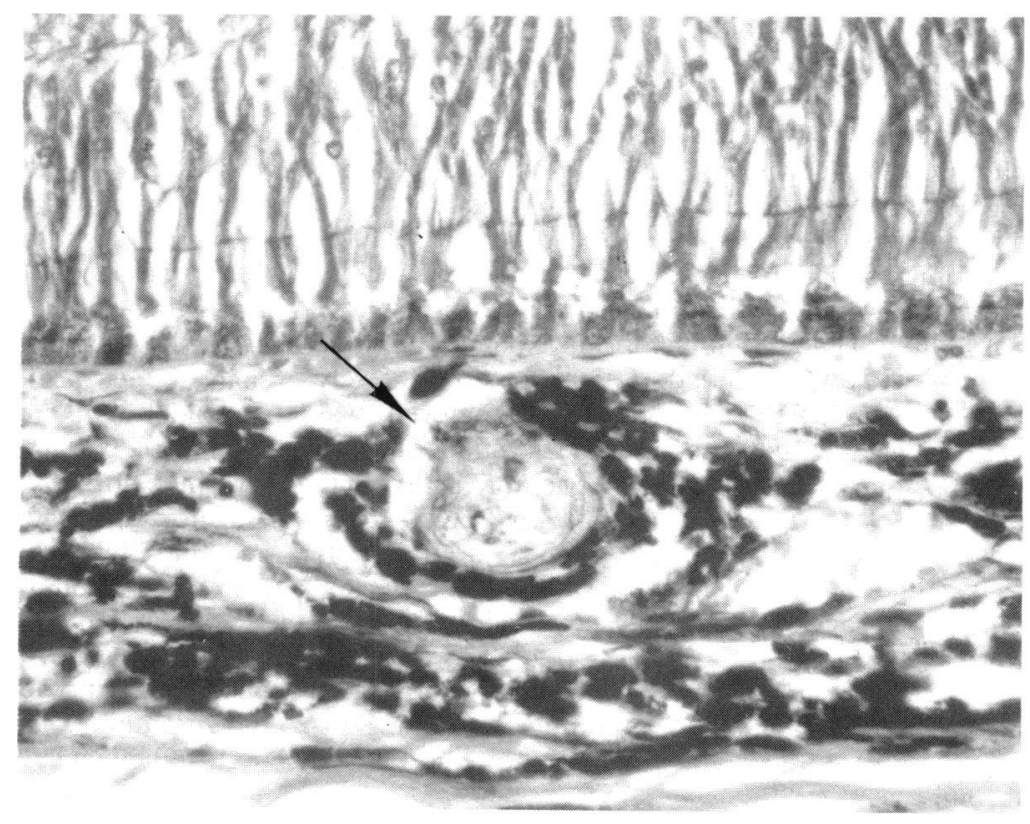


also occur after surgery to fatty areas, such as the breast."

In Purtscher's' original report all the patients had sustained head trauma. He suggested that trauma to the head was transmitted by the cerebrospinal fluid around the optic nerves with resultant stasis and passage of perivascular lymph into the retina.

Marr and Marr ${ }^{2}$ in their review noted that sudden elevation of intrathoracic pressure caused by direct trauma or deceleration forces could produce the picture of Purtscher's retinopathy. They postulated that this was due to increased venous back pressure causing haemorrhage, small vessel occlusion, and retinal oedema.

Several antecedent injuries producing sudden elevation in intravascular pressure have been described. Fundus changes were followed as they evolved in a pilot who ejected from his jet travelling at supersonic speed. ${ }^{12}$ One hour after injury there was arteriospasm with minimal haemorrhage but with visual acuity of counting fingers. The following day there were definite haemorrhages, both flame shaped and round, along with cotton-wool patches. By the following day all findings were more pronounced, with subhyaloid haemorrhages. On the fourth day oedema was more generalised, giving the macula a cherry-red appearace. However, his vision did improve. Slowly the haemorrhages and oedema resolved, with the patient regaining normal visual acuity and fundus appearance after 3 months.

Gass ${ }^{6}$ considered that Purtscher's retinopathy from chest compression is probably caused by capillary damage. Fluorescein angiography in the patient he described showed collapse and late leakage of the capillary bed in the area of the optic nerve, where the haemorrhages and exudates were. His patient later developed optic atrophy.

Kelley ${ }^{7}$ was able to examine a patient within an hour of chest compression by a lap and shoulder seat belt in a car accident. Fundus abnormalities, including superficial exudates, perimacular oedema, and haemorrhages, were evident in the right eye within an hour of the accident. But fluorescein angiography done within 2 hours of injury showed leakage from small vessels in both eyes. Unlike the patient reported by Gass, ${ }^{6}$ this patient had no areas of nonperfusion. Kelley ${ }^{7}$ favoured the possibility of direct damage to the capillary endothelium as most likely, although he believed microemboli could not be ruled out as a cause. His patient's vision returned to normal within 2 weeks, but the fundus appearance did not resolve until 10 weeks after the injury.

Some patients with a typical Purtscher's retinopathy sustained minor trauma, such as a fall from a bicycle or gymnastic equipment, without the loss of consciousness. ${ }^{2}$ The authors suggested that such injuries might result in a transient rise of the venous pressure within the head. The retinopathy in Purtscher's patients may be explained on the same basis.

Marr and Marr ${ }^{2}$ pointed out that it can be difficult to separate the retinopathy of fat embolism from that of increased hydrostatic pressure, but thought that this can be done in part by the presence or absence of a fat-generating injury, such as a fracture, and associated systemic findings, such as pulmonary and cerebral involvement. In their opinion fat emboli produce retinopathy at least a day or two after the event, while in retinopathy from increased hydrostatic pressure the fundus findings appear within hours. They believed diffuse oedema, with a cherryred appearance of the macula, is more probably due to fat emboli.

Duane ${ }^{13}$ reported several patients with history of trauma and varying fundus pictures. He believed that the retinopathy classically called Purtscher's was caused by combined rapid rises of arterial and venous pressures. A combination of hard exudates, inner retinal haemorrhages, and arterial spasms were caused by arterial pressure rise, and retinal oedema, transudation, and preretinal haemorrhage were caused by venous pressure rise. He believed the fundus findings were too variable, with injuries sometimes too complex, to be ascribed simply to angiospasm or fat emboli.

Other hypotheses, by no means mutually exclusive, include air emboli ${ }^{14}$ and granulocyte aggregates secondary to complement activation. ${ }^{4}$

Burton ${ }^{14}$ thought air embolism to be an alternative possibility in chest compression injuries. He cited experimental work in which animals that died of chest compressive injuries had air emboli in the lungs; however, the eyes were not examined. Burton ${ }^{14}$ also pointed out in his follow-up of 4 patients that fine pigmentary changes occurred in the posterior poles of 2 of them, suggesting that choroidal as well as retinal embolism had taken place.

It has been observed that acute pancreatitis can be associated with a similar retinopathy. ${ }^{3}$ The authors presented 3 patients with pancreatitis who had blurred vision during the disease course. Two patients had fluorescein angiograms which showed areas of arteriolar and capillary nonperfusion and late leakage, consistent with numerous small emboli. The authors stated that fat emboli from omental adipose tissue, digested by enzymes released from the inflamed and damaged pancreas, were responsible for the eye findings, as fat emboli to other organs in pancreatitis had been demonstrated. ${ }^{3}$

In a more recent report of a fourth patient with pancreatitis and Purtscher's retinopathy ${ }^{4}$ the authors speculated that, rather than emboli of fat, the emboli 
were aggregates of granulocytes caused by activated C5, a component of complement. The authors could not directly prove that the patient's retinopathy was due to aggregates of granulocytes or that in their patient complement C5 levels were elevated. However, they obtained acute and convalescent serum from 12 other patients admitted to hospital with pancreatitis, and showed that their serum would aggregate granulocytes in the acute stage of the disease but not after convalescence. They were able to demonstrate that either trypsin or activated C5 added to normal serum would also cause aggregation.

Experimental work in vitro has shown that complement factor $\mathrm{C5}$, when activated, was the responsible factor in granulocyte aggregation, since anti-C5 antibody abolished all aggregation activity. ${ }^{15}$ Furthermore the size of the aggregates would approximate that of the $15-40 \mu \mathrm{m}$ glass spheres used by Ashton and Henkind ${ }^{16}$ to produce a fundus appearance similar to Purtscher's retinopathy.

The authors ${ }^{4}$ proposed that digestive enzymes are released from the pancreas when it is acutely inflamed and activate complement factor $\mathrm{C} 5$, which in turn aggregates granulocytes. This theory is also consistent with Purtscher's retinopathy from trauma, because acute trauma has been shown to activate several complement factors. ${ }^{17}$ This would be another possible cause for the fundus appearance. It is quite likely that a combination of several factors, including hydrostatic pressure changes, fat emboli, air emboli, and granulocyte emboli, could be responsible.

Histopathologically we were able to demonstrate vascular occlusion at the posterior pole both in the choroid and in the retina of our patient. Because she died 23 days after admission, and 21 days from the time the retinopathy was first observed, it was not possible to characterise the emboli. Either lipid emboli or granulocytic aggregates are possible alternatives.
This study was supported in part by Research Grant 1 R01 EY-01684 from the National Eye Institute (Dr Green) and a grant from the Heed Foundation (Dr Kincaid).

\section{References}

1 Purtscher $O$. Noch unbekannte Befunde nach Schädeltrauma. Ber Dtsch Ophthalmol Ges 1910; 36: 294-307.

2 Marr WG. Marr EG. Some observations on Purtscher's disease: traumatic retinal angiopathy. Am J Ophthalmol 1962; 54: 693-705.

3 Inkeles DM, Walsh. JB. Retinal fat emboli as a sequela to acute pancreatitis. Am J Ophthalmol 1975; 80: 935-8.

4 Jacob HS, Goldstein IM, Shapiro I, Craddock, PR. Hammerschmidt DE, Weissmann G. Sudden blindness in acute pancreatitis. Possible role of complement-induced retinal leukoembolization. Arch Intern Med 1981; 141: 134-6.

5 Urbanek J. Über Fettembolie des Auges. Albrecht von Graefes Arch Klin Ophthalmol 1934; 131: 147-73.

6 Gass JDM. Atlas of Macular Disease. Diagnosis and Treatment. 2nd ed. St Louis: Mosby, 1977: 316-7.

7 Kelley JS. Purtscher's retinopathy related to chest compression by safety belts. Fluorescein angiographic findings. Am J Ophthalmol 1972; 74: 278-83.

8 Fritz MH. Hogan MJ. Fat embolization involving the human eve. Am J Ophthalmol 1948; 31: 527-34.

9 DeVoe AG. Ocular fat embolism. A clinical and pathologic report. Arch Ophthalmol 1950; 43: 857-63.

10 Kearns TP. Fat embolism of the retina demonstrated by a flat retinal preparation. Am J Ophthalmol 1956; 41: 1-2.

11 Morgan OG. Some cases of fat embolism of the retina. Trans Ophthalmol Soc UK 1949; 69: 441-5.

12 Lyle DJ. Stapp JP. Button RR. Ophthalmologic hydrostatic pressure syndrome. Am J Ophthalmol 1957; 44: 652-7.

13 Duane TD. Valsalva hemorrhagic retinopathy. Am J Ophthalmol 1973; 75: 637-42.

14 Burton TC. Unilateral Purtscher's retinopathy. Ophthalmologv 1980; 87: 1096-105.

15 Craddock PR. Hammerschmidt D. White JG. Dalmasso AP. Jacob HS. Complement C5a-induced granulocyte aggregation in vitro. A possible mechanism of complement-mediated leukostasis and leukopenia. J Clin Invest 1977; 60: 260-4.

16 Ashton N. Henkind P. Experimental occlusion of retinal arterioles using graded glass ballotini. Br J Ophthalmol 1965: 49: 225-34.

17 Ruddy S. Gigli I. Austen KF. The complement system of man. N Engl J Med 1972; 287: 489-9.5. 